\title{
Electrospray Ion Beam Deposition: Soft-Landing and Fragmentation of Functional Molecules at Solid Surfaces
}

\author{
Stephan Rauschenbach, ${ }^{+, *}$ Ralf Vogelgesang, ${ }^{\dagger}$ N. Malinowski, ${ }^{+, \perp}$ Jürgen W. Gerlach, ${ }^{*}$ Mohamed Benyoucef, ${ }^{+, \|}$ \\ Giovanni Costantini, ${ }^{+, \pi}$ Zhitao Deng, $^{+}{ }^{+}$Nicha Thontasen, ${ }^{+}$and Klaus Kern ${ }^{+, \S}$ \\ ${ }^{\dagger}$ Max-Planck-Institute for Solid State Research, Heisenbergstr. 1, DE-70569 Stuttgart, Germany, ${ }^{\ddagger}$ Leibniz-Institute for Surface Modification (I0M), Permoserstr. 15, \\ DE-04303 Leipzig, Germany, and I Institut de la Matière Condensée, Ecole Polytechnique Fédérale de Lausanne, CH-1015 Lausanne, Switzerland. ${ }^{\perp}$ Current address: \\ Central Laboratory of Photographic Processes, Bulgarian Academy of Sciences, 1113 Sofia, Acad. G. Bonchev St., Bl. 109, Bulgaria. "Current address: Institute for \\ Integrative Nanosciences, IFW Dresden, Helmholtzstrasse 20, D-01069 Dresden, Germany. "Current address: Department of Chemistry, University of Warwick, Gibbet Hill \\ Road, Coventry, CV47AL, United Kingdom.
}

$\mathrm{V}$ acuum-based deposition is one of the key technologies needed to produce surface coatings for a wide variety of applications. Although often more cumbersome than processing at ambient conditions (e.g., solution processing), vacuum deposition allows the ultimate tunability of many essential processing parameters, for instance, substrate temperature, deposition rate, or deposition amount. This results in unsurpassed control over the structure, chemical state, and morphology of the final surfaces. The conventional way to produce thin films under vacuum passes through the sublimation and the subsequent condensation of the deposited material. Such vacuum processing, however, is often impossible for large molecules and nanoparticles because of their thermal instability or their low vapor pressure. This represents a major drawback for many applications where the use of complex functional molecular units would be beneficial. In general, large molecules or nanoparticles are stable in vacuum, but the controlled in situ fabrication of a surface coating is problematic due to difficulties in bringing them into the gas phase.

The discovery of electrospray ionization $(E S I)^{1,2}$ and matrix-assisted laser desorption ionization (MALDI) $)^{3}$ made nonvolatile molecules available in the gas phase as molecular ions. ESI and MALDI ion sources are widely applied in mass spectrometric analysis, providing an essential tool for the identification of biological molecules, particularly proteins. ${ }^{4}$

With these ion sources available, ion beam deposition (IBD) becomes a viable al-
ABSTRACT The ion beam deposition (IBD) of rhodamine dye molecules on solid surfaces in high vacuum is explored in order to characterize the possibility of fabricating molecular coatings or nanostructures from nonvolatile molecules. Molecular ion beams with a well-defined composition are deposited on silicon oxide surfaces with a controlled kinetic energy. Photoluminescence spectroscopy and time-of-flight secondary ion mass spectrometry (TOF-SIMS) are employed in order to characterize the sample with respect to coverage, homogeneity, and the fraction of intact landed ions (soft-landing ratio). We find that homogeneous rhodamine films of defined composition can be produced at energies of $2-100 \mathrm{eV}$. The coverage is found to be proportional to the ion dose. Soft-landing is observed for energies up to $35 \mathrm{eV}$.

KEYWORDS: electrospray · ion beam deposition - soft-landing · rhodamine - mass spectrometry - molecular ions · fragmentation - SIMS · photoluminescence · fluorescence

ternative for the modification of surfaces under well-defined high- or ultrahigh vacuum (UHV) conditions, in particular when conventional techniques such as evaporation or chemical vapor deposition fail to produce intact gas-phase molecules or particles. Among such cases are molecular species that are promising building blocks for complex nanoscale architecture ${ }^{5,6}$ and for single-molecule electronics. ${ }^{7-10}$ For these molecules, the IBD approach enables in situ access to ultrasensitive, vacuum-based surface science techniques such as scanning tunneling microscopy (STM), photoelectron spectroscopy, or electron diffraction.

Soft-landing, the destruction-free ion beam deposition of molecular ions, ${ }^{11}$ has been the topic of numerous studies, ${ }^{12-14}$ most of them aimed at the purification or enrichment of very rare and nonvolatile organic or biological compounds. ${ }^{15,16}$ In all of those experiments, it has been demonstrated that it is actually possible to bring
*Address correspondence to s.rauschenbach@fkf.mpg.de.

Received for review January 4, 2009 and accepted September 3, 2009.

Published online September 23, 2009.

$10.1021 / \mathrm{nn} 900022$ p CCC: $\$ 40.75$

C 2009 American Chemical Society 
nonvolatile compounds from a solution or matrix into the gas phase and to deposit them on a surface, ${ }^{17,18}$ where they are present as molecular ion or as neutralized species. ${ }^{19}$ Among the molecules and particles that have been successfully deposited or soft-landed from ion beams under vacuum conditions are small organic molecules, ${ }^{11,15,16,20-23}$ proteins and peptides, ${ }^{12,14,24,25}$ whole viruses, ${ }^{13,26}$ as well as metallic and semiconducting nanoparticles. ${ }^{24,27}$

The target substrates for deposition are typically metallic or semiconducting, but also self-assembled monolayers (SAM) ${ }^{14}$ and even liquid surfaces ${ }^{16}$ have been used. It should be noted that IBD is still largely an academic research tool and the few existing experimental setups are home-built machines of vastly different designs. Due to the fact that the goal of IBD experiments is often the separation and enrichment of mass-selected compounds, extensive characterization via mass spectrometry is typical, while properties such as homogeneity and coverage of the collected material have usually received less attention. Therefore, results concerning these properties are often not directly comparable across different experiments, especially in cases where the scope of the characterization of the deposited material and the beam fabrication and characterization are not consistent.

For the vacuum deposition of nonvolatile molecules and particles, only a few alternatives to IBD exist. Pulsed valves, ${ }^{28-30}$ thermal shock evaporation, ${ }^{31,32}$ and stamp techniques ${ }^{33}$ have been demonstrated in the last years. Typically, these techniques are relatively easy to implement and do provide surface coatings in UHV, yet the resulting films often lack homogeneity, purity, control, or reproducibility.

In order to study the feasibility of IBD as a method for surface modification, we use our ion beam deposition setup, which has been designed for general application with nonvolatile materials. ${ }^{24}$ Fluorescent molecules of the rhodamine family are used to create the ion beams for deposition. Besides the importance of these dye molecules for applications in optics ${ }^{34-36}$ and life sciences, ${ }^{37}$ their strong fluorescence makes them an excellent probe for soft-landing, which can be studied ex situ after the deposition. Thereby, possible changes in the molecular structure upon incidence can be reflected in the fluorescence response, for example, in an intensity suppression or in a shift of the emitted wavelength's center.

Molecular ion beams of rhodamine are created by an electrospray ion source. These beams are characterized by in situ time-of-flight (TOF) mass spectrometry. Mass selective ion optics are used to ensure the discrimination of the undesired species, resulting in a chemically controlled beam which is then deposited at a predetermined kinetic energy on a substrate in high vacuum.
After deposition, the samples are analyzed ex situ by scanning photoluminescence (PL) spectroscopy and time-of-flight secondary ion mass spectroscopy (TOF-SIMS). The combination of these two methods allows a quantitative determination of the amount of material being soft-landed on the surface and its spatial distribution. SIMS measurements provide information whether the deposited molecule is present at the surface in intact or fragmented form. From the relative abundance of the molecular and fragment species in SIMS, the soft-landing ratio, defined as the ratio of intact ions to the total amount of deposited ions, can be determined. Photoluminescence spectroscopy measurements are performed in order to verify the characteristic fluorescence properties of the molecular species (spectral signature and photobleaching). With this approach, we are able to study the relation of the fluorescent intensity and the deposited dose which allows conclusions about the coverage. Lateral scanning measurements allow assessing the spatial homogeneity of the molecular films.

(a)

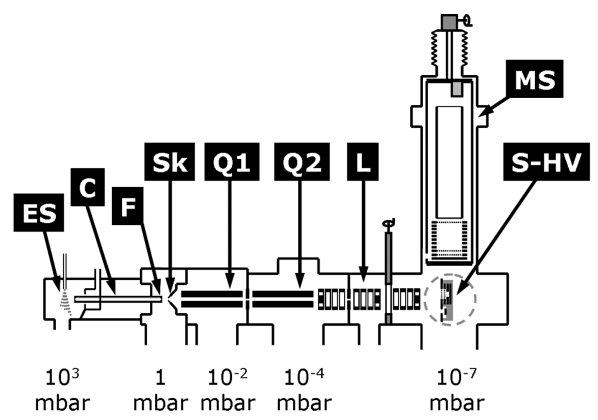

(b) front view

(c)

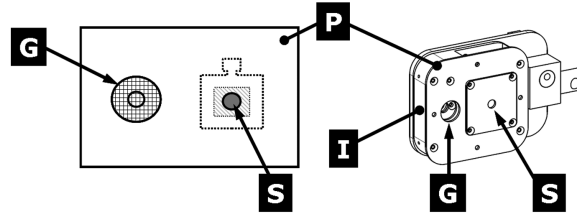

(d) cross section

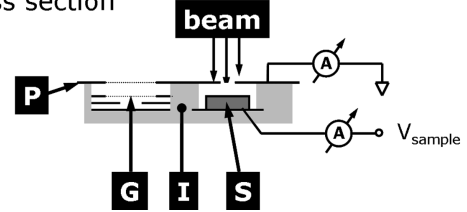

Figure 1. (a) Scheme of the IBD experiment. The pressures in the four differential pumping stages are given. ES, electrospray ion source; $C$, capillary; $F$, nozzle/fragmentor electrode; Sk, skimmer; Q1, collisional focusing RF-only quadrupole; Q2, mass selecting quadrupole; $L$, electrostatic lens; MS, time-of-flight mass spectrometer; S-HV, sample holder in high vacuum. $(b-d)$ Scheme of the high vacuum sample holder: G, retarding grid detector; $P$, cover plate; $S$, sample (behind cover plate aperture); I, insulator. The current measurement principle is indicated in (d). The voltage $V_{\text {sample }}$ is applied to the deposition substrate to adjust the incidence energy of the ion beam. 


\section{RESULTS}

The Ion Beam Deposition Experiment. The IBD experiment (Figure 1) consists of an electrospray ion source coupled to a differentially pumped vacuum apparatus in which the ion beam is formed, mass analyzed, mass selected, and deposited onto a substrate (see ref 24 for details on the apparatus). In brief, the ion beam is formed in the first three pumping stages: ions generated by the electrospray (ES) enter the vacuum through a capillary (C) and undergo a supersonic expansion upon leaving the nozzle (F) due to the pressure difference. The ion beam is skimmed (skimmer Sk) and collimated in the second pumping stage by collisional focusing in an RF-only quadrupole (Q1) operated at 0.01-0.2 mbar. Finally, a second quadrupole $(\mathrm{Q} 2)$ in the third pumping stage at $10^{-5}$ to $10^{-4} \mathrm{mbar}$ is used to select the mass range of interest for the IBD. Further focusing and guiding of the ion beam is done by electrostatic lenses (L), which include a set of steering plates each intended to adjust the beam direction.

The orthogonal extraction linear time-of-flight mass spectrometer (TOF-MS) in the fourth pumping stage (3 $\times 10^{-7} \mathrm{mbar}$ ) can detect ions in a mass-to-charge ratio range of $1-10$ Th with a mass resolution of $m / \Delta m \approx 350$ and a dynamic range spanning up to four decades. ${ }^{38}$ The main purpose of the TOF-MS in the IBD apparatus is the characterization of the ion beam before deposition in order to ensure that the beam consists only of the desired ion species. This instrument can also be used to detect characteristic fragments of molecular ions created by nozzle-skimmer fragmentation. This process may take place in the first pumping stage if the declustering potential, defined as the difference between the nozzle $(F)$ and the skimmer (Sk) voltages, is high enough to cause the rupture of intramolecular bonds due to collisions of the energetic ions with the background gas. ${ }^{39}$ Therefore, throughout the text, the nozzle will also be referred to as fragmentor electrode and the applied voltage as fragmentor voltage $\left(U_{\text {frag }}\right)$. The obtained fragmentation records provide a basis for comparison with mass spectra obtained by ex situ TOF-SIMS.

The ion deposition takes place in the fourth pumping stage. For this process, the mass spectrometer (MS) is moved upward out of the beam axis and is replaced by a sample holder (S-HV). In this assembly, the sample (S) is mounted behind an aperture of $4.5 \mathrm{~mm}$ diameter in the cover plate $(\mathrm{P})$. Moreover, the sample holder integrates a retarding grid $(G)$ detector. After the kinetic energy has been characterized, ${ }^{24}$ the ion beam is aligned with the aperture in the cover plate using the steering plates in the preceding electrostatic lens, directing the ions onto the sample surface (see Figure 1). The ion beam currents reaching the sample $(S)$ and the cover plate $(P)$ can be continuously monitored, and the ion incidence energy can be adjusted by applying a voltage to the sample $\left(V_{\text {sample }}\right.$ in Figure 1$)$. Under the assumption

\section{(a) Rhodamine 6G}
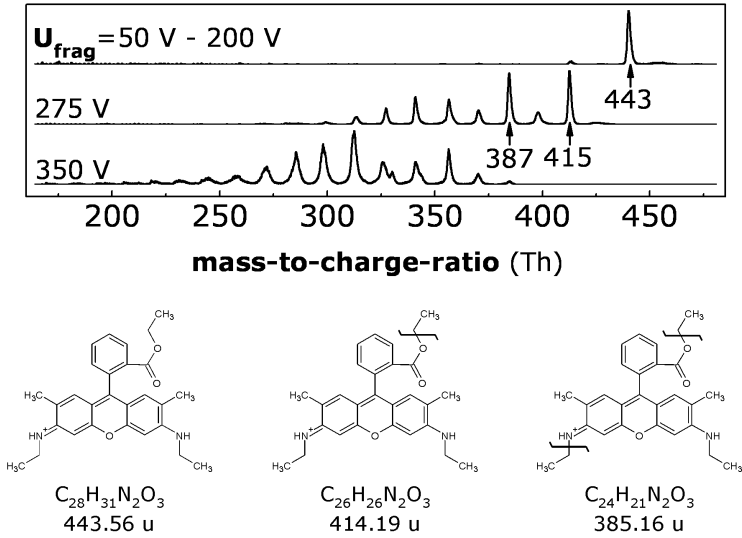

\section{(b) Rhodamine B}
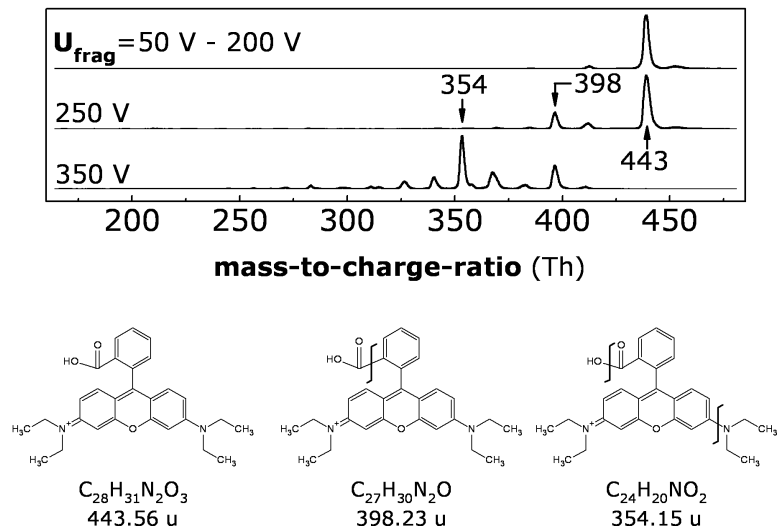

Figure 2. ESI-TOF mass spectra of Rho6G (a) and RhoB (b) for several fragmentor voltages. Below the mass spectra, the structure of the intact molecule cation and one possibility for each of the first two major fragments is displayed.

that all incident ions stick to the surface, the ion current measurement allows the amount of deposited material to be calculated if the charge state of the deposited molecules is known.

TABLE 1. Deposition Parameters of IBD Experiments with Rho6G for TOF-SIMS Analysis ${ }^{a}$

\begin{tabular}{lccccc}
$\begin{array}{c}\text { ion } \\
\text { energy (eV) }\end{array}$ & $\begin{array}{c}\text { ion beam current, } \\
\mathbf{m i n}-\max (\mathbf{p A})\end{array}$ & $\begin{array}{c}\text { duration } \\
(\mathbf{m i n})\end{array}$ & $\begin{array}{c}\text { total dose } \\
\boldsymbol{D}_{\text {tot }}(\mathbf{p A h})\end{array}$ & $\begin{array}{c}\text { coverage } \\
\left(\mathbf{1 0}^{\mathbf{3}} \boldsymbol{\mu \mathbf { m } ^ { - 2 } )}\right.\end{array}$ & $\begin{array}{c}\text { coverage } \\
(\mathbf{a r e a} \%)\end{array}$ \\
\hline 2 & $5-7$ & 175 & 18.0 & 25.5 & 2.6 \\
5 & $5-6$ & 165 & 15.0 & 21.2 & 2.1 \\
9 & $8-9$ & 155 & 15.0 & 21.2 & 2.1 \\
24 & $7-8$ & 120 & 15.0 & 21.2 & 2.1 \\
35 & $21-31$ & 30 & 13.0 & 18.4 & 1.8 \\
47 & 23 & 30 & 11.5 & 16.2 & 1.6 \\
60 & 27 & 30 & 13.3 & 18.8 & 1.9 \\
75 & $7-10$ & 135 & 17.0 & 24.1 & 2.4 \\
100 & 12.7 & 60 & 12.7 & 18.0 & 1.8 \\
5 (frag) & 15 & 60 & 15.0 & 21.2 & 2.1 \\
\hline
\end{tabular}

aThe critical parameters incidence energy, current (minimal and maximal), and duration of the deposition process are given together with the ensuing total dose $D_{\text {tot }}$ (measured in picoampere hours, $1 \mathrm{pAh}=2.25 \times 10^{-10} \mathrm{e}$ ). The calculated coverage is also displayed as number density and, for orientation, as percentage of covered surface area assuming a flat arrangement and close packing of the molecules at the surface. 


\begin{tabular}{|c|c|c|c|c|c|c|c|}
\hline $\begin{array}{c}\text { ion } \\
\text { energy }(e V)\end{array}$ & $\begin{array}{c}\text { ion beam current, } \\
\min -\max (\mathrm{pA})\end{array}$ & $\begin{array}{c}\text { duration } \\
\text { (min) }\end{array}$ & $\begin{array}{l}\text { total dose } \\
D_{\text {tot }}(\mathrm{pAh})\end{array}$ & $\begin{array}{c}\text { total } \\
\text { dose (norm) }\end{array}$ & $\begin{array}{l}\text { fluorescence } \\
\text { yield (norm) }\end{array}$ & $\begin{array}{c}\text { coverage } \\
\left(10^{3}{\left.\mu \mathrm{m}^{-2}\right)}^{-2}\right.\end{array}$ & $\begin{array}{l}\text { coverage } \\
\text { (area\%) }\end{array}$ \\
\hline 12 & $6.0-2.2$ & 20 & 1.5 & 1.00 & 1.00 & 2.1 & 0.21 \\
\hline 12 & $0.5-2.0$ & 84 & 1.3 & 0.89 & 0.87 & 1.8 & 0.18 \\
\hline 12 & $1.0-1.7$ & 70 & 1.2 & 0.63 & 0.8 & 1.7 & 0.17 \\
\hline 12 & 0.80 & 10 & 0.13 & 0.09 & 0.09 & 0.18 & 0.02 \\
\hline 18 & $19-9$ & 60 & 10.0 & & & 14.1 & 1.4 \\
\hline 10 & $2-3$ & 270 & 11.3 & & & 16.0 & 1.6 \\
\hline
\end{tabular}

aShown are incidence energy, minimal and maximal current, and duration of the deposition process together with the corresponding total ion dose $D_{\text {tot }}$ (measured in pAh). To facilitate comparison, the fluorescence intensity and the total dose are normalized to the values of the first sample, the one with the maximal coverage. The first four samples were measured with an identical set of spectrometer parameters to allow a meaningful comparison of the PL intensities with the total deposited charge (total dose).

In contrast to conductive samples, the use of insulating $200 \mathrm{~nm}$ thick $\mathrm{SiO}_{x}$ substrates for some of the IBD experiments precludes a direct measurement of the deposition current. In these cases, first the net current is determined when the ion beam hits only the cover plate. The current is then measured again after the ion beam has been deflected onto the deposition spot using the steering plates in the lens preceding the sample holder. The ion flux on the sample is calculated as the difference between these two currents.

In general, stable currents of 1-50 pA of singly charged rhodamine ions can be obtained for several hours. The kinetic energy per unit charge is measured by a retarding grid detector biased against ground. Depending on the beam adjustment, the ion energy relative to device ground is typically found between 18 and $30 \mathrm{eV}$ with a distribution width of $2 \mathrm{eV}$. Moreover, the beam energy is found to be constant during the deposition. For the experiments presented here, voltages between +23 and $-75 \mathrm{~V}$ have been applied to the samples, resulting in ion incidence energies between 2 \pm 2 and $100 \pm 2 \mathrm{eV}$ per charge at the sample. Details of the ion beams used for deposition are given in Table 1 and Table 2.

Observations. ESI-TOF Mass Spectrometry. The first step of every ion beam deposition experiment is the generation and characterization of the ion beam. Figure 2 shows ESI-TOF mass spectra for Rho6G and RhoB at some characteristic fragmentor voltages $U_{\text {frag. }}$. The two molecules are isomers $\left(\mathrm{C}_{28} \mathrm{H}_{31} \mathrm{~N}_{2} \mathrm{O}_{3} \mathrm{Cl}\right)$, which results in both having the same base peak at $443 \mathrm{Th}$ representing the molecular cation in positive mass spectra at low fragmentor voltages $U_{\text {frag }}=50-200 \mathrm{~V}$. Higher values of $U_{\text {frag }}$ result in characteristic fragment peaks. The two most intense fragment peaks for Rho6G are observed at 415 and 387 Th, while RhoB shows intense peaks at 398 and 354 Th. Beams of Rho6G generated at $U_{\text {frag }}<200 \mathrm{~V}$ are used for IBD of intact molecules, while for the deposition of fragments, beams created at $U_{\text {frag }}=250-400 \mathrm{~V}$ are employed. The mass range lower than $200 \mathrm{Th}$ might contain solvent residues and is therefore cut off by the low pressure quadrupole (Q2) operated in RF- only mode at a high voltage amplitude..$^{24,40}$ In this mode of operation a big $\mathrm{m} / \mathrm{z}$ window is selected for transmission. This procedure results in a highly pure beam containing more than $95 \%$ of the desired ion species, intact rhodamine ions or rhodamine fragments, respectively, at intensities which are sufficient for deposition.

Time-of-Flight Secondary lon Mass Spectrometry (TOF-SIMS). Samples of $\mathrm{Rho6G}$ prepared by IBD on $\mathrm{SiO}_{x}$ at incidence energies of 2-100 eV are analyzed by static TOF-SIMS measurements (Table 1 and Figure 4). For comparison, reference measurements of samples prepared by drop casting Rho6G and $\mathrm{RhoB}$ on $\mathrm{SiO}_{x}$ substrates are performed (Figure 3). For the drop-coated reference samples of both substances, the lower mass-to-charge ratio range of up to 250 Th is nearly identical (upper panel of Figure 3). Typical for SIMS in this $m / z$ range, intense peaks that can be related to individual atoms from the $\mathrm{SiO}_{x}$ substrate are observed along with less intense peaks corresponding to cluster ions formed during the $\mathrm{Ga}^{+}$bombardment. ${ }^{41}$ The two most intense peaks at 28 and 45 Th correspond to the ${ }^{28} \mathrm{Si}^{+}$ion and the ${ }^{28} \mathrm{Si}^{16} \mathrm{OH}^{+}$cluster ion, respectively. They are evident indicators of the presence of a silicon oxide surface and of adsorbed water molecules. Further, a variety of cluster ions are observed. These clusters consist of at-

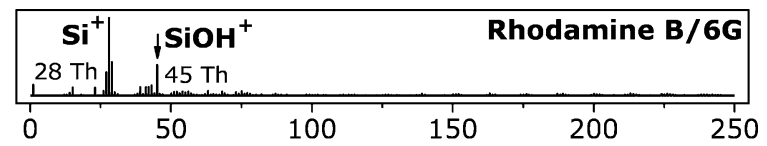

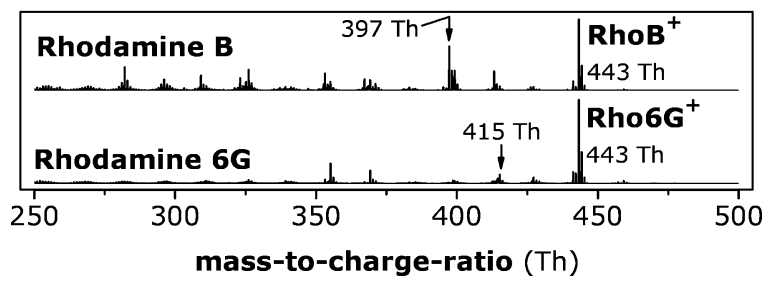

Figure 3. TOF-SIMS of reference Rho6G and RhoB samples prepared by drop casting on $\mathrm{SiO}_{x}$ substrates. The low $\mathrm{m} / \mathrm{z}$ range (upper panel) is almost identical for the two samples. In the high $\mathrm{m} / \mathrm{z}$ range (lower panel), the base peak at 443 Th corresponds to the intact molecule, while peaks at lower masses are related to rhodamine fragments or clusters generated by $\mathrm{Ga}^{+}$ irradiation. 
oms of all present species and of molecular fragments at random composition.

A small amount of contamination is found due to the fact that the samples were carried from the ion beam deposition to the TOF-SIMS characterization through atmosphere. Most pronounced are $\mathrm{Na}^{+}$at 23 Th and carbon hydrates $\mathrm{C}_{x} \mathrm{H}_{y}$ at multiples of 13,14 , or 15 Th. Because SIMS is an extremely sensitive technique, these contaminations are always found if a sample is handled in air and can only be avoided by in situ UHV sample preparation and characterization.

The lower panel in Figure 3 shows the higher massto-charge ratio range of the SIMS mass spectra (250-500 Th) obtained from drop-coated samples. In contrast to the low mass range, the spectra of Rho6G and RhoB are quite distinct from each other. The rhodamine isomer base peaks at 443 Th are the most prominent in both the Rho6G and the RhoB mass spectra, besides a hardly noticeable background of heavy clusters generated by SIMS. Both base peaks at $443 \mathrm{Th}$ are accompanied by secondary peaks of lower $\mathrm{m} / \mathrm{z}$ ratio with a characteristic spacing of $13-15$ Th, related to rhodamine fragments created by $15 \mathrm{keV} \mathrm{Ga}^{+}$irradiation of the SIMS analysis beam. For Rho6G, only a few of those peaks exceed the cluster background, while for RhoB, a large number of intense fragment peaks appear, among which the peak at 397 Th is most prominent.

Rho6G samples prepared by IBD exhibit qualitatively similar TOF-SIMS spectra as the drop cast reference samples, albeit, depending on the deposition energy, different relative peak intensities are observed (Figure 4). Peaks in the higher mass-to-charge ratio range are related to the intact molecule or to its fragments exceeding the cluster background. In particular, the most prominent peak is found at 443 Th for all samples except for the fragment deposition sample and the sample prepared at $100 \mathrm{eV}$ incidence energy, where the peak at 415 Th dominates. The samples produced at 35-100 eV incidence energy show an additional intense peak at 415 Th, as well.

Photoluminescence. The photoluminescence (PL) spectra of Rho6G deposited on $200 \mathrm{~nm} \mathrm{SiO}_{x}$ by IBD show the broad fluorescence signature between 500 and 600 $\mathrm{nm}$ that is characteristic of intact Rho6G (see Figure $5 a)$. The overall intensity of this feature decreases with measurement time due to photobleaching, a typical effect that accompanies the fluorescence of organic emitters. It confirms that rhodamine $6 \mathrm{G}$ is indeed observed. The intensity integrated over the spectral feature, relative to the excitation photon flux, is used as a measure of the uniformity of the spatial distribution as well as to compare the amount of material deposited on different samples.

Spatial scans of the PL yield across two samples with different coverage are shown in Figure 5b. The fluorescent signal is nearly constant across the depos-

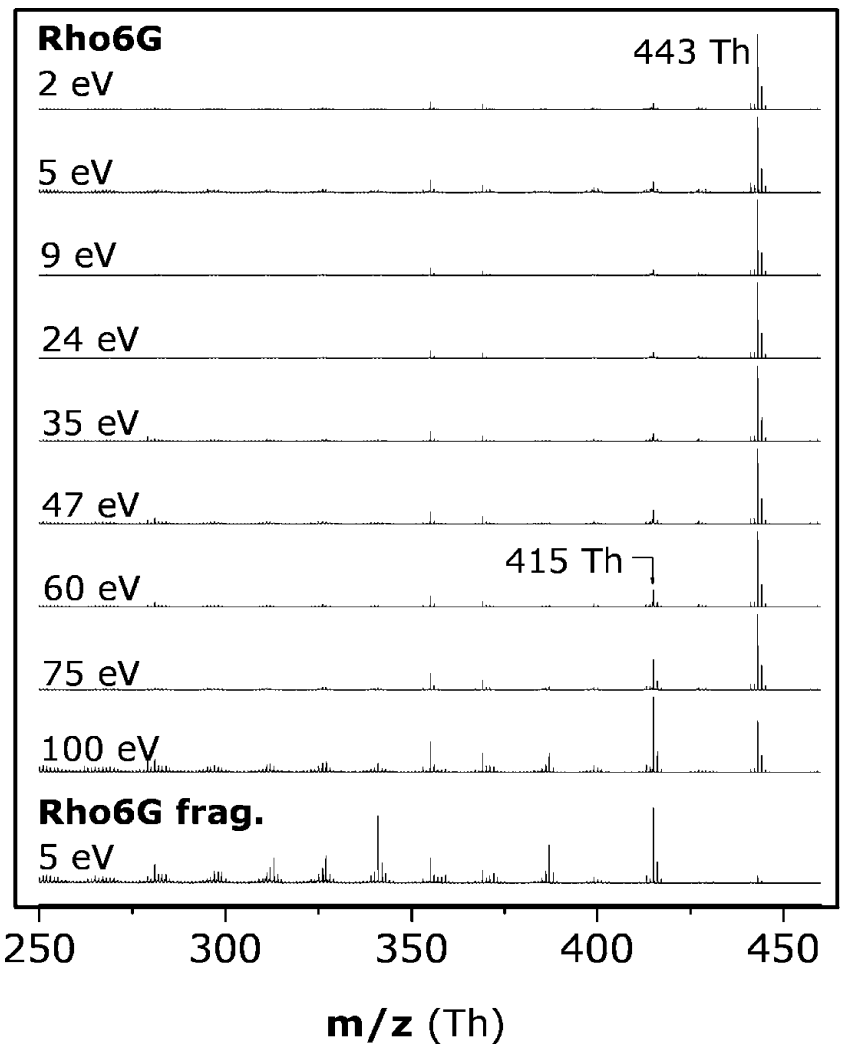

Figure 4. TOF-SIMS of Rho6G and Rho6G fragments on native $\mathrm{SiO}_{x}$ substrates deposited by IBD at incidence energies of 2-100 eV. Only the high mass-to-charge ratio range is displayed. For IBD of intact Rho6G, the peaks at 443 Th dominate the spectra up to $75 \mathrm{eV}$, and above this energy, 415 Th becomes predominant. The fragment at 415 Th has a significantly increased intensity for ion incidence energies above $35 \mathrm{eV}$. The spectra of samples prepared by depositing intentionally produced rhodamine fragments show intense peaks corresponding to the composition of the fragment ion beam used for deposition (compare with Figure $2 \mathrm{a}$ at high values of $U_{\text {frag }}$ ).

ited spot, which is $4.5 \mathrm{~mm}$ in diameter and corresponds to the size of the deposition aperture in the sample holder (Figure 1). In Table 2, the observed fluorescence intensities are compared with the total ion dose derived from the ion current measurement during deposition. Values for fluorescence intensity and deposited dose, normalized to the maximum, are given for samples that (a)

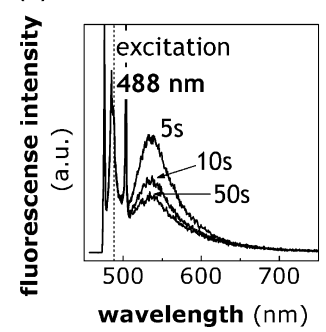

(b)

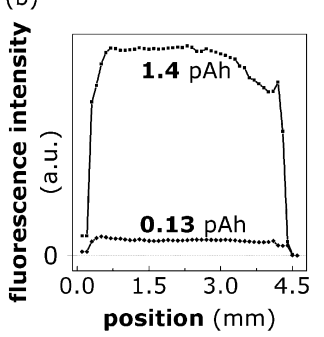

Figure 5. (a) Room temperature photoluminescence spectra of Rho6G on $\mathrm{SiO}_{x}$. The characteristic broad Rho6G fluorescence feature is observed between 500 and $600 \mathrm{~nm}$. The dashed line indicates the excitation wavelength. Photobleaching (the exposure time is indicated) leads to reduced PL yield. (b) Photoluminescence intensity across the sample acquired by lateral scans for two different coverages (dose given in $\mathrm{pAh}$ ). The luminescence intensity is integrated over the wavelength range of $500-600 \mathrm{~nm}$. 
were measured with the same settings of the PL spectrometer and can thus be quantitatively compared. Both numbers represent a relative quantitative measurement of the coverage after the ion beam deposition. Although being derived from two independent types of measurements, these values are clearly in good agreement with each other. The determined coverage values (see Table 1 and Table 2) correspond to a few percent of a monolayer. At such low coverage, the fluorescence yield collected from the surface is proportional to the amount of deposited material.

\section{DISCUSSION}

The presented IBD scheme allows complete control of the deposition process at all stages. The ion beam current, kinetic energy, and composition are adjustable and can be monitored by an ESI-TOF-MS and a retarding grid current detector in the IBD chamber. Most notably, it ensures a deposition with an ion beam that consists entirely of intact Rho6G ions - free of contamination by solvent molecules or fragmented species. Control measurements show that no neutrals are reaching the substrate surface (see Materials and Methods for more detail). Thus, on the sample, one only finds Rho6G from the ion beam or products of subsequent reactions, for instance, upon collision with the surface.

Structural differences of the two isomers Rho6G and $\mathrm{RhoB}$ are reflected in different fragmentation mass spectra both in ESI-TOF-MS and in TOF-SIMS. In both cases, the most abundant peaks form a characteristic pattern (Figures 2 and 3). The specific characteristics of these fragmentation patterns depend on the particular mass spectrometric technique. Therefore, only a partial match is observed between the corresponding spectra. This is due to the differences in the fragmentation mechanism and environment. In the case of the reference RhoB sample, the most intense peaks in TOF-SIMS are 443, 397, and 354 Th (Figure 3). The latter fragments are abundant also in ESI-TOF-MS fragmentation mass spectra (Figure 2b). On the contrary, the fragmentation pattern observed in ESI-TOF-MS for Rho6G (Figure $2 \mathrm{a}$ ) is not reproduced by TOF-SIMS to that large extent (Figure 3).

Instead, fragment peaks of the reference Rho6G in the TOF-SIMS spectra never exceed $10 \%$ of the base peak intensity, which allows for an easy interpretation of the Rho6G mass spectra observed with TOF-SIMS after IBD. In fact, the occurrence of peaks with significantly higher intensity relative to the molecular peak at 443 Th can be correlated to a different origin than the fragmentation by $\mathrm{Ga}^{+}$bombardment, assuming that drop casting deposits only intact molecules on the surface.

The result of a TOF-SIMS analysis is strongly influenced by the chemical environment at the surface. With respect to that, the two deposition techniques,
IBD and liquid deposition, can be considered as very similar for a $\mathrm{SiO}_{x}$ surface. In both cases, the rhodamine 6G is present as molecular cation accompanied by nearby counterions. The $\mathrm{Cl}^{-}$counterion is delivered to the surface by the liquid deposition together with Rho6G. In the other case, electronegative terminal oxygens at the $\mathrm{SiO}_{x}$ surface provide a similar surrounding. The binding state of the rhodamine ion is thus comparable since, in both cases, the rhodamine ion is interacting with its neighbors via ionic or ion-dipole interactions that are generally weaker than the covalent bonds within the molecule itself.

In the case of the IBD experiment, fragments can be generated by surface-induced dissociation due to the energetic impact of Rho6G ions during deposition. In order to determine the extent of this dissociation, static TOF-SIMS measurements of the IBD samples are analyzed as a function of deposition energy, in particular in the high mass-to-charge ratio range. At the $\mathrm{SiO}_{x}$ surface, the binding state of the fragment cations is the same as for the intact landed molecules. Thus secondary ion yields of both species are comparable, and the observed quantities can be directly related to coverage ratios.

The probability that an intact Rho6G is fragmented by $\mathrm{Ga}^{+}$ions during SIMS is, to a high extent, independent of the technique by which the molecule has been deposited on the surface. As a consequence, the observation of an identical ratio between the 443 and the 415 Th peaks for the low energy IBD and drop-casted samples is a first indication that Rho6G molecules remain intact during IBD deposition up to $35 \mathrm{eV}$ incidence energy.

The soft-landing ratio, $\eta$, is defined as the ratio between the number of intact molecules, $N^{*}$, and the total number, $N$, of molecules deposited by IBD, thus $\eta=N^{*} / N$. A quantitative evaluation of $\eta$ can be deduced from the intensity of the molecular and fragment peaks of the TOF-SIMS spectra in the high mass range. Essential for this analysis is the consideration that, at the deposition energy of 2-100 eV, the major fraction of the incoming beam sticks to the surface, even if the collision event results in fragmentation. ${ }^{14,42,43}$ Even though most molecules are heavily fragmented by the energetic $\mathrm{Ga}^{+}$analysis beam, the original ratio of intact to non-intact molecules should be preserved because the survival probability upon $\mathrm{Ga}^{+}$impact should be similar for high mass fragments like 415 Th and the intact Rho6G. ${ }^{44}$ Since also the ionization cross section can be assumed to be similar, $\eta$ can be determined by the relative peak intensities of the TOF-SIMS mass spectra in the high $\mathrm{m} / \mathrm{z}$ range.

The TOF-SIMS spectra of the reference drop-coated Rho6G samples show a ratio $N_{415} / N_{443}$ of less than 0.1 , where $N_{415}$ and $N_{443}$ are the integrated intensities of the first two characteristic rhodamine peaks at 443 and 415 Th, respectively. According to this observation, a 


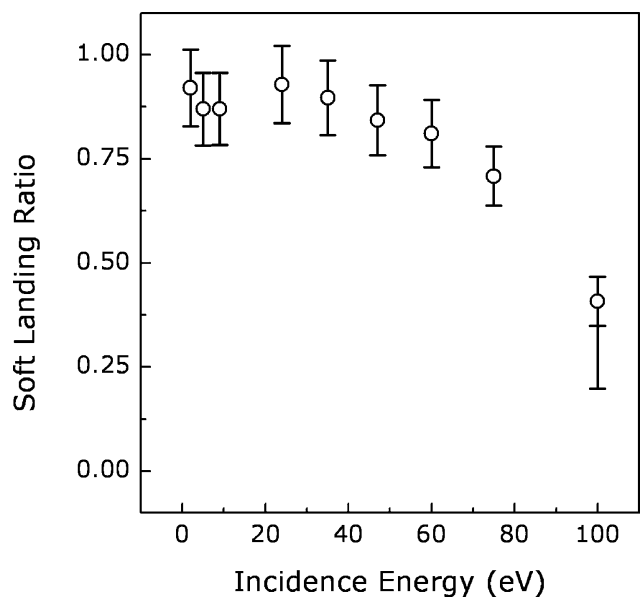

Figure 6. Soft-landing ratio as a function of incidence energy calculated from the high $\mathrm{m} / \mathrm{z}$ range of the TOF-SIMS data.

relative error in the intensity of $10 \%$ is assumed and the soft-landing ratio is calculated as $\eta=N^{*} / N=$ $N_{443} /\left(N_{443}+N_{415}+N_{387}+\cdots\right)$ and displayed in Figure 6 as a function of the incidence energy.

An almost constant value close to unity can be found for energies up to $37 \mathrm{eV}$, decreasing slowly and reaching 0.73 at the energy of $75 \mathrm{eV}$. For higher values, more fragments than intact molecules are observed in TOF-SIMS and, correspondingly, a soft-landing ratio below 0.5 is found at $100 \mathrm{eV}$ incidence energy. A larger error bar has been considered for this value since the background base signal in the corresponding TOF-SIMS mass spectra is much increased. Furthermore, for these high values of kinetic energy, a unity sticking probability of all molecules and fragments deposited cannot be assumed any more. Similar behavior of the softlanding ratio has been observed for the deposition of peptide ions on self-assembled monolayers. ${ }^{45}$

For a molecule to be soft-landed, the kinetic energy must dissipate during the collision process, without breaking of covalent bonds. The energy must therefore be distributed into vibrational and electronic degrees of freedom of molecule and substrate. Thereby, none of the excited degrees of freedom must exceed a certain fragmentation threshold. For metallic nanoparticles, an estimate of the soft-landing limit has been found experimentally and by simulations to scale with the size of the cluster as $1 \mathrm{eV} /$ atom. ${ }^{46,47}$ Similar energies have been observed for the soft-landing of complex molecules of different size. ${ }^{14,19,20,25}$ In the case of rhodamine $6 \mathrm{G}$, the $1 \mathrm{eV} /$ atom estimate results in a softlanding threshold of $64 \mathrm{eV}$. This agrees well with the energies for which we measure a decreasing soft-landing ratio. However, the mechanisms involved in softlanding of molecules are still a field of lively research. ${ }^{14,48}$ Future research with the IBD scheme demonstrated here will allow more elaborate measurements of the soft-landing ratio. In addition, the possibil- ity to couple UHV-based surface-sensitive analytics in situ will add information about the involved processes.

Previous ion beam deposition experiments already employed optical detection schemes such as fluorescence microscopy ${ }^{20,25}$ or surface-enhanced Raman spectroscopy ${ }^{18}$ to identify and quantify the amount of deposited material. In particular, fluorescent molecular ions from an electrospray ion beam have been deposited on a metal surface with a high flux, and the amount of material was determined by fluorescent spectrometry of the washed-off molecules in solution. ${ }^{20}$

In our experiments, the comparison of the relative values for the coverage obtained by ion current integration and by PL shows a good agreement. In general, an absolute quantification of the amount of deposited material from the fluorescence yield is possible only after a suitable calibration against a known coverage standard. However, our measurements demonstrate that the ion current recorded during deposition proves to be just as reliable for the determination of the coverage, in particular, since the charge state is typically known after ESI-TOF mass spectrometry. While the indirect discrimination of intact molecules and their fragments by analysis of optical spectroscopic signatures may be possible, SIMS provides more immediate, chemically specific information.

\section{CONCLUSION}

The result of the electrospray ion beam deposition (ES-IBD) at the given energies is complete or partial softlanding of rhodamine molecules as a homogeneous layer across the whole deposition spot, which is shown by fluorescence detected in the PL measurements in combination with static TOF-SIMS. The measurements show that the amount of material detected is proportional to the deposited charge and homogeneously distributed over a macroscopic length scale of several millimeters. Moreover, the applicability of photoluminescence spectrometry for the quantification of the surface coverage after IBD as well as the reproducibility of the deposition technique is demonstrated. IBD with in situ ESI-TOF-MS characterization, energy, and current measurement now allows the full control of the deposition process on a solid surface.

Our ion beam deposition setup has been developed to meet the requirements of in situ UHV analysis of the deposited molecules. On one side, this demands the preparation of atomically defined solid surfaces and the possibility of their in situ characterization. On the other side, a deposition technique is needed that provides a controlled surface modification, as it is the case with ES-IBD. The presented setup has recently been extended by two further vacuum stages and a link to an organic MBE system, allowing a deposition at $10^{-10}$ mbar onto a solid surface that is prepared and checked by in situ STM at the same base pressure. The coverages demon- 
strated in this paper can be produced in a reasonable time of several hours ranging from densities of single molecules in the typical STM scan area of 100 $\times 100 \mathrm{~nm}^{2}$ up to full monolayers.

Such molecular systems are ideal candidates for investigations with surface science techniques, in particular, using scanning probe instruments. A very low coverage can be used to investigate the molec- ular shape and electronic structure of molecules preferably at cryogenic temperatures, ${ }^{49}$ and higher coverage of up to a full monolayer can be used when supramolecular assemblies are to be studied. ${ }^{5}$ Ion beam deposition thus opens the way to investigate the intrinsic properties of functional nonvolatile molecules and their reactions at surfaces under perfectly controlled conditions.

\section{MATERIALS AND METHODS}

Molecules for the Ion Beam. The dye molecules rhodamine 6G (Rho6G) and rhodamine B (RhoB) (see Figure 2) are commercially available (Sigma Aldrich R4127 and R6626, respectively). Thermal decomposition studies show that above $500 \mathrm{~K}$ both molecules decompose during evaporation..$^{50}$ The same study also indicates that below that temperature the intact molecule is evaporated. However, there are a few applications of rhodamine $6 \mathrm{G}$ sublimation in vacuum, ${ }^{51,52}$ indicating that the sublimation can easily be accompanied by thermal decomposition of the molecule.

Both molecules dissolve easily in a mixture of methanol (analytical grade) and water. Formic acid (analytical grade) is used to acidify the solution. Typically, between 0.1 and $1.0 \mathrm{vol} \%$ is added, which boosts the ion yield but does not affect the observed mass spectra. The ion beams for deposition are created by electrospray ionization of these mixtures at concentrations of $0.2-2.0 \mu \mathrm{mol} / \mathrm{mL}$ and are further characterized and tuned before deposition.

Deposition Substrates. Silicon wafers with native or thermally grown oxide layers are used as deposition substrates. A thermally grown oxide layer of $200 \mathrm{~nm}$ helps avoiding fluorescence quenching near the conductive Si surface and is therefore used for the photoluminescence spectroscopy measurements. Silicon surfaces with a native oxide (typically a few nanometers thick) are, on the contrary, utilized for TOF-SIMS experiments. The insulating nature of these substrates does not affect the ion beam deposition due to Coulomb repulsion of the deposited charges, as long as the coverage is well below a monolayer. ${ }^{14}$

Ion Beam Deposition. The total deposited dose $D_{\text {tot }}$ is given by

$$
D_{\text {tot }}=\int_{t=0}^{t_{\text {depo }}} I_{\text {depo }}\left(t^{\prime}\right) \mathrm{d} t^{\prime}
$$

is calculated directly from the deposition current $I_{\text {depo }}(t)$ and deposition time $t_{\text {depo }}$ and thus has the dimension of a charge. The current is recorded during the experiment at several electrodes including the sample. In Table 1 and Table 2, the integrated total doses are given together with the minimum and maximum currents measured throughout the experiment.

In order to keep the experimental conditions comparable during the deposition of different samples, we refrain from readjusting the ion beam after each deposition. This results in the ion current being different for each sample (see Table 1 and Table 2) due to effects such as drift of power supplies or changed position of the sample holder.

Control experiments for neutral contamination are performed by applying a voltage of $60 \mathrm{~V}$ to the sample before a beam of fragments is directed to it. That voltage is sufficiently larger than the ion beam energy and thus should repel all of the incoming rhodamine fragment ions. In fact, the ion current observed on the sample becomes zero. Under these conditions, only neutral molecules or lowly charged droplets of high kinetic energy are able to reach the substrate surface. However, this is avoided by placing the sample intentionally off the beam axis and steering the beam to the deposition spot via the steering plates in the lens upstream the sample holder. After such a treatment, no rhodamine molecules nor fragments are found at the surface if analyzed with TOF-SIMS.
TOF-SIMS Reference Samples. Reference samples for TOF-SIMS measurements were produced by immersing clean Si samples in a $10 \mu \mathrm{M}$ solution of rhodamine in water and ethanol (1:1) for several seconds. After taking out, all solution remains are blown off with dry nitrogen. The rhodamine film of samples produced in this way is not visible to the naked eye. However, the coverage has not been determined in further detail.

Photoluminescence Spectroscopy. For this study, two equivalent setups were used to detect the fluorescence signal of rhodamine on the $200 \mathrm{~nm} \mathrm{SiO}_{x}$ samples. Both systems employ laser excitation, notch filters to separate the elastic component from the fluorescence signal, and multichannel spectrometers to detect the optical spectrum.

In the first case, photoluminescence is excited and spectra are collected with a confocal microscope (Leica TCS SP2), dispersed in an F/4 monochromator (ACR 300i) and recorded with a 1024 channel CCD detector (PI-MAX) operating at $235 \mathrm{~K}$.

In the second setup, micro-photoluminescence measurements are performed at room temperature using a continuous wave frequency-doubled $\mathrm{Nd}: \mathrm{YVO}_{4}$ laser operating at $532 \mathrm{~nm}$. The sample is mounted on a motorized $x y$-linear translation stage whose motion is computer-controlled for exact positioning with a precision of $50 \mathrm{~nm}$. The laser beam is focused by a $20 \times$ microscope objective on the sample surface to a spot diameter of about $5 \mu \mathrm{m}$. The PL signal is collected with the same microscope objective and is spectrally filtered by a $0.75 \mathrm{~m}$ focal length spectrometer equipped with a liquid nitrogen cooled CCD detector.

Time-of-Flight Secondary Ion Mass Spectrometry (TOF-SIMS). Rhodaminecovered samples were analyzed by secondary ion mass spectrometry performed on a TOFSIMS 5 (IonTOF GmbH, Münster, Germany) time-of-flight reflectron spectrometer. The instrument was operated in static mode using a $15 \mathrm{keV} \mathrm{Ga}^{+}$ion beam for the characterization of the substrate surface. Positive ion mass spectra are accumulated for $500 \mathrm{~s}$ from an area of $500 \times 500 \mu \mathrm{m}^{2}$ over which the $\mathrm{Ga}^{+}$beam is scanned. Samples analyzed by TOFSIMS have been exposed to air for several days during transfer.

Acknowledgment. The authors thank Dr. K. Amsharow and Dr. T. Weitz for the fruitful discussions and the critical reading of the manuscript. Prof. Dr. O. Schmidt is acknowledged for the access to his optics laboratory, and Dr. U. Starke and T. Acartürk for performing additional TOF-SIMS measurements. We further thank the reviewers for their comments and suggestions, which helped us to improve the original manuscript. S.R. was supported by the Hans-L.-Merkle Foundation for excellence in science and technology.

\section{REFERENCES AND NOTES}

1. Fenn, J. B.; Mann, M.; Meng, C. K.; Wong, S. F.; Whitehouse, C. M. Electrospray lonization for Mass-Spectrometry of Large Biomolecules. Science 1989, 246, 64-71.

2. Yamashita, M.; Fenn, J. B. Electrospray lon-SourceAnother Variation on the Free-Jet Theme. J. Phys. Chem. 1984, 88, 4451-4459.

3. Karas, M.; Bachmann, D.; Bahr, U.; F., H. Matrix-Assisted Ultraviolet-Laser Desorption Of Nonvolatile Compounds. Int. J. Mass Spectrom. Ion Processes 1987, 78, 53-68. 
4. Smith, R. D.; Loo, J. A.; Loo, R. R. O.; Busman, M.; Udseth, H. R. Principles and Practice of Electrospray lonizationMass-Spectrometry For Large Polypeptides and Proteins. Mass Spectrom. Rev. 1991, 10, 359-451.

5. Barth, J. V.; Costantini, G.; Kern, K. Engineering Atomic and Molecular Nanostructures at Surfaces. Nature 2005, 437, 671-679.

6. Wessendorf, F.; Hirsch, A. Self-Assembly of Supramolecular Oligo-Phenylene-Ethynylene Wires Consisting of Double Hamilton Receptor Modified OPE Rods and a Tetraphenylporphyrin Cyanurate. Tetrahedron 2008, 64, 11480-11489.

7. Vives, G.; Carella, A.; Launay, J.-P.; Rapenne, G. A StarShaped Ruthenium Complex with Five FerrocenylTerminated Arms Bridged by Trans-Platinum Fragments. Chem. Commun. 2006, 2283-2285.

8. Rajadurai, C.; Schramm, F.; Brink, S.; Fuhr, O.; Ghafari, M.; Kruk, R.; Ruben, M. Spin Transition in a Chainlike Supramolecular Iron(II) Complex. Inorg. Chem. 2006, 45, 10019-10021.

9. Mayor, M.; Didschies, C. A Giant Conjugated Molecular Ring. Angew. Chem., Int. Ed. 2003, 42, 3176-3179.

10. Payer, D.; Rauschenbach, S.; Konuma, M.; Virojanadara, $C_{.}$ Starke, U.; Dietrich, C.; Collin, J., J. P.; Sauvage; Lin, N.; Kern, K. Toward Mechanical Switching of Surface-Grafted [2]Catenante by In-Situ Copper Complexation. J. Am. Chem. Soc. 2007, 129, 15662-15667.

11. Franchetti, V.; Solka, B. H.; Baitinger, W. E.; Amy, J. W.; Cooks, R. G. Soft Landing of Ions as a Means of Surface Modification. Int. J. Mass Spectrom. Ion Processes 1977, 23 29-35.

12. Ouyang, Z.; Takats, Z.; Blake, T. A.; Gologan, B.; Guymon, A. J.; Wiseman, J. M.; Oliver, J. C.; Davisson, V. J.; Cooks, R. G. Preparing Protein Microarrays by SoftLanding of Mass-Selected lons. Science 2003, 301, 1351-1354.

13. Siuzdak, G.; Bothner, B.; Yeager, M.; Brugidou, C.; Fauquet, C. M.; Hoey, K.; Chang, C. M. Mass Spectrometry and Viral Analysis. Chem. Biol. 1996, 3, 45-48.

14. Laskin, J.; Wang, P.; Hadjar, O. Soft-Landing of Peptide lons onto Self-Assembled Monolayer Surfaces: An Overview. Phys. Chem. Chem. Phys. 2008, 10, 1079-1090.

15. Volny, M.; Turecek, F. High Efficiency in Soft Landing of Biomolecular lons on a Plasma-Treated Metal Surface: Are Double-Digit Yields Possible? J. Mass Spectrom. 2006, 41, 124-126.

16. Gologan, B.; Takats, Z.; Alvarez, J.; Wiseman, J. M.; Talaty, N.; Ouyang, Z.; Cooks, R. G. lon Soft-Landing into Liquids: Protein Identification, Separation, and Purification with Retention of Biological Activity. J. Am. Soc. Mass Spectrom. 2004, 15, 1874-1884.

17. Feng, B. B.; Wunschel, D. S.; Masselon, C. D.; Pasa-Tolic, L.; Smith, R. D. Retrieval of DNA Using Soft-Landing after Mass Analysis by ESI-FTICR for Enzymatic Manipulation. J. Am. Chem. Soc. 1999, 121, 8961-8962.

18. Volny, M.; Sengupta, A.; Wilson, C. B.; Swanson, B. D.; Davis, E. J.; Turecek, F. Surface-Enhanced Raman Spectroscopy of Soft-Landed Polyatomic lons and Molecules. Anal. Chem. 2007, 79, 4543-4551.

19. Gologan, B.; Green, J. R.; Alvarez, J.; Laskin, J.; Cooks, R. G. Ion/Surface Reactions and lon Soft-Landing. Phys. Chem. Chem. Phys. 2005, 7, 1490-1500.

20. Volny, M.; Elam, W. T.; Ratner, B. D.; Turecek, F. Preparative Soft and Reactive Landing of Gas-Phase lons on PlasmaTreated Metal Surfaces. Anal. Chem. 2005, 77, 4846-4853.

21. Satterley, C. J.; Perdigao, L. M. A.; Saywell, A.; Magnano, G.; Rienzo, A.; Mayor, L. C.; Dhanak, V. R.; Beton, P. H.; O'Shea, J. N. Electrospray Deposition of Fullerenes in Ultra-High Vacuum: In-Situ Scanning Tunneling Microscopy and Photoemission Spectroscopy. Nanotechnology 2007, 18, 455304.

22. Mayor, L. C.; Taylor, J. B.; Magnano, G.; Rienzo, A.; Satterley, C. J.; O'Shea, J. N.; Schnadt, J. Photoemission, Resonant Photoemission, and X-ray Absorption of a Ru(II) Complex
Adsorbed on Rutile $\mathrm{TiO}_{2}$ (110) Prepared by In-Situ Electrospray Deposition. J. Chem. Phys. 2008, 129, 114701.

23. Rader, H. J.; Rouhanipour, A.; Talarico, A. M.; Palermo, V.; Samori, P.; Mullen, K. Processing of Giant Graphene Molecules by Soft-Landing Mass Spectrometry. Nat. Mater. 2006, 5, 276-280.

24. Rauschenbach, S.; Stadler, F. L.; Lunedei, E.; Malinowski, N.; Koltsov, S.; Costantini, G.; Kern, K. Electrospray Ion Beam Deposition of Clusters and Biomolecules. Small 2006, 2, 540-547.

25. Volny, M.; Elam, W. T.; Branca, A.; Ratner, B. D.; Turecek, F. Preparative Soft and Reactive Landing of Multiply Charged Protein lons on a Plasma-Treated Metal Surface. Anal. Chem. 2005, 77, 4890-4896.

26. Fuerstenau, S. D.; Benner, W. H.; Thomas, J. J.; Brugidou, C.; Bothner, B.; Siuzdak, G. Mass Spectrometry of an Intact Virus. Angew. Chem., Int. Ed. 2001, 40, 982.

27. Tani, Y.; Kobayashi, S.; Kawazoe, H. Characterization of Electrospray lon-Beam-Deposited CdSe/ZnS Quantum Dot Thin Films From a Colloidal Solution. J. Vac. Sci. Technol., A 2008, 26, 1058-1061.

28. Yamada, T.; Suzuki, H.; Miki, H.; Maofa, G.; Mashiko, S. HighResolution Scanning Tunneling Microscopy Images of Molecular Overlayers Prepared by a New Molecular Beam Deposition Apparatus with Spray-Jet Technique. J. Phys. Chem. B 2005, 109, 3183-3188.

29. Okabayashi, Y.; Mitarai, T.; Yamazaki, S.; Matsuyama, R.; Kanai, K.; Ouchi, Y.; Seki, K. Preparation and Characterization of Rhodamine B and Alq3 Thin Films Deposited by Solution Jet Beam Method. Appl. Surf. Sci. 2005, 244, 217-220.

30. Okabayashi, Y.; Kanai, K.; Ouchi, Y.; Seki, K. Apparatus for Solution Jet Beam Deposition of Organic Thin Films and In-Situ Ultraviolet Photoelectron Spectroscopy. Rev. Sci. Instrum. 2006, 77, 033905.

31. Beuhler, R. J.; Flanigan, E.; Greene, L. J.; Friedman, L. Proton Transfer Mass Spectrometry of Peptides. Rapid Heating Technique for Underivatized Peptides Containing Arginine. J. Am. Chem. Soc. 1974, 96, 3990-3999.

32. Zambelli, T.; Jiang, P.; Lagoute, J.; Grillo, S. E.; Gauthier, S.; Gourdon, A.; Joachim, C. Deformation of a 3.7-nm Long Molecular Wire at a Metallic Step Edge. Phys. Rev. B 2002, 66, 075410.

33. Vitali, L.; Fabris, S.; Mosca Conte, A.; Brink, S.; Ruben, M.; Baroni, S.; Kern, K. Electronic Structure of SurfaceSupported Bis(phthalocyaninato)terbium(III) Single Molecular Magnets. Nano Lett. 2008, 8, 3364.

34. Magde, D.; Wong, R.; Seybold, P. G. Fluorescence Quantum Yields and Their Relation to Lifetimes of Rhodamine $6 \mathrm{G}$ and Fluorescein in Nine Solvents: Improved Absolute Standards for Quantum Yields. Photochem. Photobiol. 2002, 75, 327-334.

35. Selanger, K. A.; Falnes, J.; Sikkeland, T. Fluorescence Lifetime Studies of Rhodamine $6 \mathrm{G}$ in Methanol. J. Phys. Chem. 1977, 81, 1960.

36. Nie, S.; Emroy, S. R. Probing Single Molecules and Single Nanoparticles by Surface-Enhanced Raman Scattering. Science 1997, 275, 1102.

37. Titus, J. A.; Haugland, R.; Sharrow, S. O.; Segal, D. M. Texas Red, a Hydrophilic, Red-Emitting Fluorophore for Use with Fluorescein in Dual Parameter Flow Microfluorometric and Fluorescence Microscopic Studies. J. Immunol. Methods 1982, 50, 193-204.

38. Cooks, R. G.; Rockwood, A. L. The Thomson-A Suggested Unit for Mass Spectroscopists. Rapid Commun. Mass Spectrom. 1991, 5, 93.

39. Schneider, B. S.; Douglas, D. J.; Chan, D. D. Y. Ion Fragmentation in an Electrospray lonization Mass Spectrometer Interface with Different Gases. Rapid Commun. Mass Spectrom. 2001, 15, 249-257.

40. Gerlich, D. Inhomogenous RF Fields: A Versatile Tool for the Study of Processes with Slow lons; John Wiley and Sons Inc.: New York, 1992.

41. Colton, R. J.; Ross, M. M.; Kidwell, D. A. Secondary lon Mass Spectrometry: Polyatomic and Molecular Ion Emission. 
Nucl. Instrum. Methods Phys. Res., Sect. B 1986, B13, 259-277.

42. Grill, V.; Shen, J.; Evans, C.; Cooks, R. G. Collisions of lons with Surfaces at Chemically Relevant Energies: Instrumentation and Phenomena. Rev. Sci. Instrum. 2001, 72, 3149-3179.

43. Cooks, R. G.; Jo, S. C.; Green, J. Collisions of Organic lons at Surfaces. Appl. Surf. Sci. 2004, 231-2, 13-21.

44. Lange, W.; Bennighoven, A.; Jirikowsky, M. Secondary lon Emission from W-IV-Deposited Amino Acid Overlayers on Metals. Surf. Sci. 1984, 136, 419-436.

45. Alvarez, J.; Futrell, J.; Laskin, J. Soft-Landing of Peptides onto Self-Assembled Monolayer Surfaces. J. Phys. Chem. A 2006, 110, 1678-1687.

46. Cheng, H. P.; Landman, U. Controlled Deposition, Soft Landing, and Glass-Formation in Nanocluster-Surface Collisions. Science 1993, 260, 1304-1307.

47. Bromann, K.; Felix, C.; Brune, H.; Harbich, W.; Monot, R.; Buttet, J.; Kern, K. Controlled Deposition of Size-Selected Silver Nanoclusters. Science 1996, 274, 956-958.

48. Nie, Z.; Li, G.; Goodwin, M. P.; Gao, L.; Cyriac, J.; Cooks, R. G. In Situ SIMS Analysis and Reactions of Surfaces Prepared by Soft Landing of Mass-Selected Cations and Anions Using an lon Trap Mass Spectrometer. J. Am. Soc. Mass Spectrom. 2009, 20, 949-956.

49. Gimzewski, J.; Joachim, C. Nanoscale Science of Single Molecules Using Local Probes. Science 1999, 283, 5408.

50. Davie, E.; Morris, J. H.; Smith, W. E. Electron-Impact and Thermal-Degradation of Rhodamine F5G Chloride and Related Compounds. Org. Mass Spectrom. 1974, 9, 763.

51. Uehara, Y.; Ushioda, S. Single Molecule Spectrum of Rhodamine 6G on Highly Oriented Pyrolytic Graphite. Appl. Phys. Lett. 2005, 86, 181905.

52. Maennig, B.; et al. Organic p-i-n Solar Cells. Appl. Phys. A: Mater. Sci. Process. 2004, 79, 1-14. 